\title{
Learning Dynamics with Private and Public Signals *
}

\author{
Adam Copeland ${ }^{\dagger}$ \\ Board of Governors of the Federal Reserve System
}

December 6, 2004

\begin{abstract}
This paper studies the evolution of firms' beliefs in a dynamic model of technology adoption. Firms play a simple variant of the classic two-armed bandit problem, where one arm represents a known, deterministic production technology and the other arm an unknown, stochastic technology. Firms learn about the unknown technology by observing both private and public signals. I find that because of the externality associated with the public signal, the evolution of beliefs under a market equilibrium can differ significantly from that under a planner. In particular, firms experiment earlier under the planner than they do under the market equilibrium and thus firms under the planner generate more information at the start of the model. This intertemporal effect brings about the unusual result that, on a per period basis, there exist cases where firms in a market equilibrium over-experiment relative to the planner in the latter periods of the model.
\end{abstract}

\footnotetext{
${ }^{*}$ I would like to thank Matt Mitchell and Tom Holmes for their advice and encouragement. I would also like to thank Thor Koeppl, Cyril Monnet, John Stevens, and Jason Cummins for their helpful comments. This paper is the second chapter of my dissertation. The views expressed herein are my own and not necessarily those of the Federal Reserve Board.

${ }^{\dagger}$ I can be reached at adam.m.copeland@frb.gov
} 


\section{Introduction}

When a new technology is introduced into the marketplace, firms typically are unsure about its true quality. Over time, firms learn through direct and indirect means how the new technology compares with the old, existing one. The technology adoption literature has studied the diffusion of information under restrictive informational constraints. Firms either all hold the same beliefs about the new technology, or they hold heterogeneous beliefs but learn and act in isolation. As shown in the herding literature however, the combination of both heterogeneous beliefs and learning from others creates interesting and potent effects on the sequential generation of information in an economy. This paper adds to the literature by constructing a dynamic model of technology adoption that incorporates both heterogeneous beliefs and social learning and by studying the speed of adoption within such an environment.

The model in this paper embeds the classic two-armed bandit problem into a finiteperiod equilibrium model of technology adoption. ${ }^{1}$ The adoption decision that firms face is to determine which technology, or arm, to use to produce output each period. One arm represents the "old" technology and has a known, deterministic output distribution common to all players. The second arm represents the "new" technology and has an unknown, stochastic output distribution common to all players. The mean output of the stochastic technology, however, can be either higher or lower than that of the deterministic technology. There are no costs to operating either technology, nor are there costs to switching from one technology to the other between periods.

This model departs from the usual two-armed bandit analysis through its informational structure. I assume that agents can observe only their own individual output and a noisy signal of aggregate output. Experimenting with the new technology and observing individual output conveys the usual learning-by-doing effect. As firms can observe only their own output, this learning-by-doing effect is a private signal that generates heterogeneous beliefs among firms. This effect encourages firms to adopt the new technology, as information has positive value.

In contrast to the individual, private aspect of experimentation, the noisy signal of

\footnotetext{
${ }^{1}$ Two good sources on one- and two-armed bandit problems are Rothschild (1974) and Berry and Fristedt (1985).
} 
aggregate output is a public signal observed by all agents. Aggregate quantity contains useful information as its value depends upon the true mean of the stochastic technology. Intuitively, a signal of unexpectedly high aggregate quantity is more likely to occur if the new, stochastic technology is superior to the old technology. Because it is observed by all firms regardless of their actions, the public signal creates an informational externality. As such, this signal generates the usual free-rider effect found in models with public signals. Unlike the typical bandit problem, however, the public signal is not an ad hoc specification but a function of the model equilibrium and the distribution of firms' beliefs. As the informational value of the public signal varies with the measure of firms that experiment with the new technology, the learning interaction among firms is tied to economic fundamentals. A key feature of the public signal is the increase in its informational value as the measure of adopting firms rises.

To solve the firm's problem in this rich informational environment, I define an anonymous sequential game. Under this equilibrium, the following cutoff rule applies. Firms holding beliefs greater than the cutoff belief adopt the stochastic technology; firms holding beliefs below the cutoff belief choose the deterministic technology; and firms holding the cutoff belief employ a mixed strategy.

To determine whether the speed with which firms adopt the stochastic technology is efficient, I define and solve the social planner's problem. Because firms hold heterogeneous beliefs and there is aggregate uncertainty, I cannot formulate a classic planner's problem that maximizes expected aggregate quantity. So instead, I define an uninformed social planner's problem, where the planner seeks to maximize each firm's expected output conditional on the firm being no worse off relative to the market equilibrium outcome. In essence, an uninformed planner coordinates firms' actions so as to implement a Pareto-optimal outcome that Pareto dominates the market outcome. The uninformed social planner is the relevant point of comparison, as this formulation maintains the same informational constraints that firms face under a market equilibrium.

Firms' expected output increases under a social planner, because the planner can coordinate firms' experimentation strategies and take full advantage of the informational externality associated with the public signal. To show that the standard result holds is easy: ceteris paribus, the planner induces a higher level of adoption among firms in a 
given period than the market equilibrium does. More interesting, this model highlights how the planner shifts the generation of information to earlier periods in the model because information is more valuable the earlier it is generated. As a result of this intertemporal substitution, the planner induces a lower level of experimentation than firms under a market equilibrium in later periods of the model. This finding is at odds with models with more restrictive informational frameworks, which predict that firms always under-experiment relative to the social planner.

The under-adoption result is driven by two separate forces. First, the informational externality of the public signal results in the planner's use of a different experimentation strategy relative to the market equilibrium. Consequently the sequential generation of information across these two regimes differs, as does, therefore, the evolution of aggregate beliefs across the two regimes. Second, the existence of private signals ensures that firms hold heterogeneous beliefs. This heterogeneity and the nonmonotonic return on information across beliefs imply that firms vary in the way they value additional information. In the extreme, firms that believe that the risky technology is good or bad with 100 percent probability do not value additional information. Hence, I can construct a case in which the social planner, relative to the market equilibrium, generates more information through higher experimentation, in the initial periods of the model. As a consequence, in the later periods of the model, firms under the planner place less value on additional information, and as a result initiate less experimentation than firms in a market equilibrium.

This paper builds on three bodies of literature. The first is the field of social learning. I use a framework close to Bolton and Harris (1999), who examine a multiperiod, multiplayer two-armed bandit problem and explore the strategic interactions among agents. As they did in their paper, I try to answer the same basic questions about the speed and diffusion of information among agents, but I do using an environment with heterogeneous beliefs and without strategic effects. I consider an economy with a measure 1 of agents, resulting in perfect competition in information, as no single agent can influence the aggregate outcome. This simplification allows for a more-complex modeling of the public signal and makes an environment with both private and public signals tractable. This additional complexity allows us to analyze the efficient dissemination of information 
in a world with heterogeneous beliefs.

Second is the literature on technology adoption under uncertainty. Using a twoarmed bandit framework where each arm has an unknown output distribution, Jensen (1983) analyzes the belief dynamics of firms when they have access to private signals. He demonstrates that a simple learning-by-doing mechanism will generate heterogeneous beliefs, resulting in an ogive-shaped diffusion curve. Unlike this paper, Jensen (1983) does not incorporate informational externalities and so predicts that firms learn efficiently. Rob (1991) establishes that, in an ad hoc model of entry, a public signal can also generate gradual learning even when beliefs are homogeneous. In addition, he shows that by internalizing the informational externality associated with the public signal, the social planner will induce a higher level of entry relative to firms in a market equilibrium, ceteris paribus. Furthermore, he proves that the planner will always choose a higher level of entry throughout all periods of the model. The present paper builds upon Rob's result by highlighting a subtle inefficiency in the rate of adoption in competitive markets - the slower generation of information. I show how the planner wants not only to induce more adoption to take advantage of the informational externality of the public signal but also to make firms adopt earlier in the model because information is more valuable the sooner it is revealed.

Third is the work on herding/information cascades by Banerjee (1992), Bikhchandani, Hirshleifer, and Welch (1992), and more recently Dasgupta (2002). As is done in the present paper, this field of research studies the sequential generation of information and

its effects on agents' adoption, or entry, decisions. In addition, the previous papers study agent behavior when both private and public signals exist. Unlike my paper, however, the herding literature focuses on certain inefficiencies in the generation of information that do not exist within this paper's framework.

\section{A Three Period Example}

In this section I describe a three period example of the model. After outlining the parameter values of the model, I intuitively describe the firm's problem and present the example's results. 


\subsection{Environment}

There is a measure 1 of firms producing one good, where each firm seeks to maximize output. The model has three periods, in each of which each firm decides which of two production technologies to use. A firm's objective is to produce the most output over all three periods. The first technology is "safe", always producing $\bar{Y}$. The second technology is "risky", producing $\mu \in\{\underline{\mu}, \bar{\mu}\}$, where $\underline{\mu}<\bar{Y}<\bar{\mu}$. The probability distribution over $\mu$ depends upon a parameter $s \in\{L, H\}$. Thus, the output of the deterministic technology is known and common to all firms, whereas the output of the stochastic technology is unknown and common to all firms. I assume that $\bar{Y}=0.5, \bar{\mu}=1$, and $\underline{\mu}=0$. I impose symmetry on the distribution of $s$, and assume that $\operatorname{Pr}(\bar{\mu} \mid s=H)=\operatorname{Pr}(\underline{\mu} \mid s=L)=0.7$. Letting $Y_{s}$ denote the conditional expected output from using the stochastic technology, the parameter choices imply that $Y_{L}=0.3$ and $Y_{H}=0.7$. In seeking to maximize output, the firm needs to determine which production technology is better. Under the chosen parameters, the safe technology is better if $s=L$ and worse if $s=H$.

There are no costs involved with production nor with switching technologies. Aggregate uncertainty is present in this environment as the parameter $s$ is unknown. This parameter has the same value over all three periods but is unobserved by the firm. Firms have priors, denoted $\gamma$, over $s \in\{L, H\}$. As $s$ can take only two values, let $\gamma=\operatorname{Pr}(s=H)$.

Firms learn about $s$ through two channels. First, they can experiment with the stochastic technology and observe their individual output. Firms only can observe their own output, and so this information is a private signal of $s$. Firms use Bayes rule to update their beliefs over $s$. Given the prior belief $\gamma$, let $\bar{\gamma}(\gamma)$ denote a firms' posterior belief after observing $\bar{\mu}$. Similarly, let $\underline{\gamma}(\gamma)$ denote a firm's posterior belief after observing $\underline{\mu}$, and note that Bayes rule implies that $\underline{\gamma}<\gamma<\bar{\gamma}$ for $\gamma \in\{0,1\}$. The second channel of learning occurs through a noisy signal of aggregate quantity, $\tilde{Q}$, which is observed at the end of the period. Regardless of their actions, all firms see the same signal, making $\tilde{Q}$ a public signal of $s$. As described in more detail later, $\tilde{Q}=Q+\varepsilon$, where $Q$ is the actual aggregate quantity produced, and $\varepsilon \sim U[-b, b]$, where $b>0$. The uniform distribution of $\varepsilon$ makes this model tractable, as it reduces the informational gain of the public signal to two extremes: either firms learn the true value of $s$ with certainty after observing $\tilde{Q}$, 
or they learn nothing after observing $\tilde{Q}$. In this example, the upper and lower bound of the uniform distribution, $b$, is equal to 0.6. This interval is a large enough to ensure that the probability of learning the true value of $s$ from the public signal, denoted $\tilde{p}$, is less than one. The probability that a firm will learn from the public signal is a function of all firms' actions. As section 3 will show, the more firms that adopt the stochastic technology, the higher the probability that $\tilde{Q}$ reveals the true value of $s$. In other words, the strength of the public signal is increasing in the measure of adopting firms.

With the public signal, the firm's problem is a mixture of learning-by-doing and social learning. The major forces at work, however, can be described intuitively by using the framework of the firm's static problem. This problem is

$$
V(\gamma)=\max _{e \in[0,1]}\left\{(1-e) \bar{Y}+e\left(\gamma Y_{H}+(1-\gamma) Y_{L}\right)\right\}
$$

where $e$ is the adoption strategy of the firm. Here, the firm compares the output from the deterministic technology against the expected output from the stochastic technology. The decision rule is a cutoff belief, above which the firm uses the stochastic technology with probability 1. At the cutoff belief, the firm is indifferent between using either technology. Below the cutoff, firms adopt the stochastic technology with probability 0.

In a multiperiod problem with learning, it is significant that $V$ is increasing and convex in $\gamma$. Attaching a signal of $s$ to the stochastic technology means that the adopting firm's next-period belief will be either $\underline{\gamma}$ or $\bar{\gamma}$. Bayes rule implies that $\underline{\gamma}<\gamma<\bar{\gamma}$ and $\underline{\gamma}[1-\alpha(\gamma)]+\bar{\gamma} \alpha(\gamma)=\gamma$, where $\alpha(\gamma)$ is the prior probability of observing $\bar{\mu}$. By Jensen's inequality, the convexity of $V$ tells us the sum of $V(\underline{\gamma})$ and $V(\bar{\gamma})$, weighted by the probability of observing $\underline{\mu}$ and $\bar{\mu}$, is greater than $V(\gamma)$; information increases the next period's expected output. Although information is valuable, its value is not monotonic in $\gamma$. Those firms that have 'strong' beliefs, with $\gamma$ close to 0 or 1 , gain less from additional information than firms with $\gamma$ close to 0.5 .

The public signal blunts the return from the stochastic technology. This signal reveals the true value of $s$ with some positive probability. Thus, the learning benefits from observing the stochastic technology accrue only when the public signal is uninformative.

Through the public signal, the adoption decisions of all firms influence the decision of an individual firm. This influence complicates the notation required to formally describe 
the firm's problem. The formal description adds little intuition, and so it appears in the latter part of the paper. Consequently, in this example I will not lay out the firm's full problem and will only touch upon the equilibrium concept used in the model.

The equilibrium concept used in this model is an anonymous sequential game (ASG) introduced by Jovanovic and Rosenthal (1988). In an ASG, all firms pursue outputmaximizing strategies that are best responses to all other firms' optimal strategies. The equilibrium is of an anonymous nature in that firms care only about the distribution of firms' beliefs, not about the individual identities of the firms on the distribution. The state space for the firm's problem, then, is the aggregate distribution of firms' beliefs. In the first period of the problem, the distribution of firms' beliefs is simply the initial prior that all firms hold, which I assume is a point mass known to all firms. The distribution of beliefs in the second period, however, depends upon all firms' actions as well as upon the value of $s$. In equilibrium, firms can rationally deduce all other firms' adoption decisions. There is, however, uncertainty over whether 70 percent of adopting firms observed $\underline{\mu}$ or $\bar{\mu}$. Conditional on $s$, all firms agree on the aggregate distribution of firms' beliefs. Firms disagree, however, on the probability that $s=H$. This disagreement can be sustained in equilibrium, as each firm is too small to affect the aggregate distribution of beliefs. As a result, the state space for the firm's problem consists of three elements: the firm's belief about the value of $s$, the distribution of firms' beliefs conditional on $s=L$ and the distribution of firms' beliefs conditional on $s=H$.

Later in the paper, I fully specify the firm's problem and fully define an ASG. Here one needs only to know that there is a unique equilibrium and that in every period the firm's policy rule is a cutoff function.

\subsection{Solving the Model}

The first step in solving the model is defining the set of feasible beliefs that firms can hold in all periods of the model. I assume that firms start the model holding the same prior belief, $\gamma=0.45$, and, for simplicity, do not discount. ${ }^{2}$

\footnotetext{
${ }^{2}$ There is a range of prior beliefs for which the main results of this paper hold. With all other parameters held fixed, the use of any prior belief with a point mass in the range of 0.442 to 0.456 will generate the main over-adoption results of this paper. In addition, the main results of this paper can
} 
Table 1: Set of Feasible Beliefs, $\operatorname{Pr}(s=H)$

\begin{tabular}{|c|c|c|}
\hline 1st Period & 2nd Period & 3rd Period \\
\hline & 1 & 1 \\
& & .82 \\
.45 & .66 & .66 \\
& .45 & .45 \\
& .26 & .26 \\
& & .13 \\
& 0 & 0 \\
\hline
\end{tabular}

As discussed before, the timing of the model is such that firms choose which technology to use, produce output, and then observe their individual output and the noisy signal of aggregate output. After observing the signals of $s$, firms update their beliefs and enter the next period. Given an initial belief, firms could hold five possible beliefs in the second period, and seven in the third. Table 1 displays the set of feasible beliefs in all three periods. At the end of the first period, all firms observe a public signal that may reveal the true value of $s$. Hence, firms' beliefs at the beginning of the second period could equal 0 or 1 . When the public signal is uninformative, the firms that adopt the stochastic technology learn about $s$ by observing their individual output. Under Bayes rule, a firm's possible posterior beliefs are either 0.66 or 0.26 , depending on whether the firm observed $\bar{\mu}$ or $\underline{\mu}$, respectively. ${ }^{3}$ The firms that use the safe, deterministic technology learn nothing and so continue to hold their prior belief of 0.45 . In the third period, the set of feasible beliefs continues to expand in this fashion. This set increases only by two elements, as under Bayes rule those firms that observe both $\bar{\mu}$ and $\mu$ end up with a posterior belief equal to their initial prior belief, regardless of the order in which those outputs are observed.

Using the set of possible beliefs and the assumed parameters, I solve the firm's problem through backward induction. Solving the firm's problem is difficult because the second period's state space, the two distributions of aggregate beliefs conditional on $s$, is be generated for the case in which firms discount the future.

${ }^{3}$ The formula used to derive these posterior beliefs is later provided in equation 5 . 
Figure 1: Second Period Distribution of Firms' Beliefs under an ASG

True Value of $s$ is Low

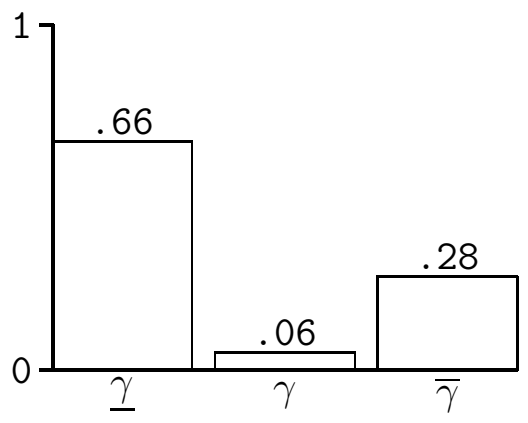

True Value of $s$ is High

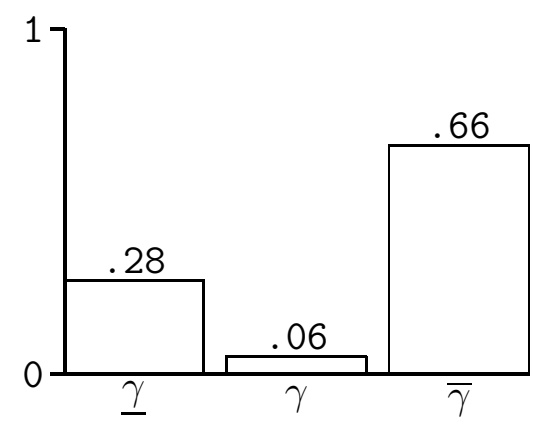

large. Standard computational techniques, however, can surmount this problem. These details are described in appendix A.1.

\subsection{Results}

The first period's cutoff belief is 0.45 , the prior belief that all firms hold. All firms employ the same mixed strategy of adopting the stochastic technology with 94 percent probability. Given an uninformative public signal, this adoption strategy results in the second-period conditional distributions of aggregate beliefs charted in figure 1. As shown in this figure, measure 0.94 of firms adopt, receive a private signal and update their prior beliefs. For the case in which $s=L, 30$ percent of adopting firms observe $\bar{\mu}$. As a result, measure $0.28=0.3 * 0.94$ of firms hold the posterior belief $\bar{\gamma}=0.66$. The remaining 70 percent of adopting firms observe $\underline{\mu}$ and hold the posterior $\underline{\gamma}=0.26$. Finally, firms that do not adopt, and so learn nothing, enter the second period with their initial prior, $\gamma=0.45$. The symmetry in the model causes the distribution of beliefs when $s=H$ to be the mirror image of the distribution of beliefs when $s=L$.

In the second period, the conditional distributions of beliefs described above result in the optimal policy rule that all firms with $\gamma>0.44$ adopt the stochastic technology. Thus all but those firms with $\gamma=0.26$ adopt the stochastic technology. As shown in table 2 , a measure of $0.34=0.06+0.28$ firms adopt the stochastic technology in the second period, given $s=L$. For $s=H$ the measure of adopting firms is $0.72=0.06+0.66$. 
Table 2: Measure of Firms Adopting

\begin{tabular}{|c|c|c|c|c|}
\hline & Period & ASG & SP & Difference \\
\hline \multirow{3}{*}{$s=L$} & 1 & .94 & 1.00 & -.06 \\
& 2 & .34 & .30 & .04 \\
& 3 & .10 & .09 & .01 \\
\hline \multirow{3}{*}{$s=H$} & 1 & .94 & 1.00 & -.06 \\
& 2 & .72 & .70 & .02 \\
& 3 & .50 & .49 & .01 \\
\hline
\end{tabular}

In the third and final period, the firm's problem reduces to a static problem. Given the symmetry of the parameter assumptions, the cutoff belief is $\gamma=0.5$. Given firms' adoption strategies in the second period, this policy rule results in measure 0.1 firms adopting the risky technology in the third period, if $s=L$. If $s=H$, measure 0.5 firms adopt.

\subsection{Social Planner's Problem}

To analyze the efficiency of firms' adoption decisions in an ASG, a comparable social planner's problem must be defined. The standard social planner's problem, however, is not applicable in this environment. As firms hold heterogeneous beliefs and the true aggregate distribution of beliefs is unknown, which belief the planner should hold and how that belief is updated are unclear. If a planner can observe all firms' individual output, then for any positive measure of adopting firms the planner will learn the true value of $s$ after one period. To make the planner's problem an interesting comparison to the firm's problem under an ASG, I define an uninformed social planner's problem. As detailed later, this type of planner's problem reduces to coordinating firms' adoption decisions so that the resulting outcome is Pareto-optimal and Pareto dominates the outcome under an ASG. Under an uninformed social planner, firms are able to pursue adoption strategies that take advantage of the externalities inherent in models with public signals. 
Figure 2: Second Period Distribution of Firms' Beliefs under an SP

True Value of $s$ Is Low

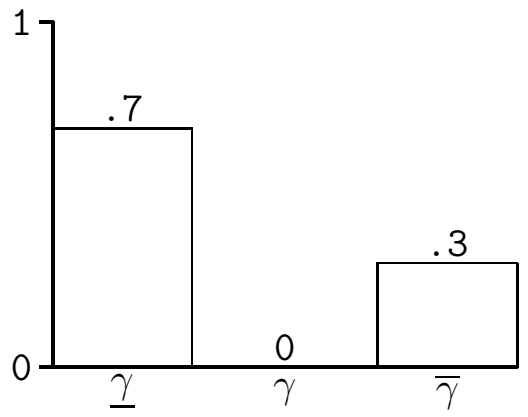

True Value of $s$ Is High

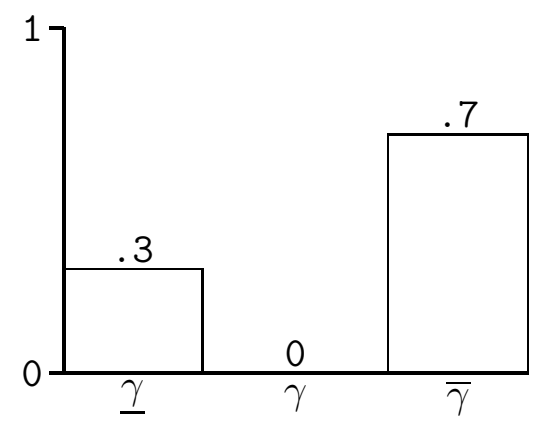

The optimal adoption strategies under this planner's problem are, as in an ASG, cutoff beliefs. As before, when firms hold the cutoff belief, their optimal strategy is a mixed strategy (see theorem 2 later in the paper). As with an ASG, I solve for the planner's adoption strategies by using backward induction. An additional twist to the planner's problem is the lack of a unique equilibrium; however, for the parameters chosen in this example, there is a unique solution. ${ }^{4}$

Under a social planner, the optimal adoption strategy is for firms to adopt the risky technology with probability 1. Assuming an uninformative public signal, this adoption strategy results in the second-period conditional aggregate distribution of beliefs charted in figure 2, which shows all firms massed at $\underline{\gamma}=0.26$ and $\bar{\gamma}=0.66$. Not surprisingly, the optimal adoption strategy in the second period is for those firms with the belief $\bar{\gamma}$ to adopt the stochastic technology with probability 1 , and for those holding $\underline{\gamma}$ to use the safe technology. As shown in table 2, this adoption strategy implies that the measure of firms adopting the risky technology in the second period is 0.3 if $s=L$. For $s=H$, this measure of adopting firms is 0.7 .

As shown in table 2, both measures of adoption are lower than those under the ASG equilibrium. Hence, the planner, in the first period, induces a higher level of experimentation in the first period, but in the second period dictates a lower level of experimentation for the case in which the public signal is uninformative. In essence,

\footnotetext{
${ }^{4}$ There is a unique solution in this example because in the first period the optimal adoption strategy is a corner solution - everyone adopts with probability one. See appendix A.1 for more details.
} 
the planner has firms experimenting earlier to generate more information about $s$ at the beginning of the model. Relative to the ASG, this earlier experimentation not only increases the strength of the public signal in the first period but also raises the variance of the second period aggregate distribution of beliefs. Under a social planner, no firms hold the initial prior - a belief where the return on observing the private signal is relatively high. As a consequence, firms in the second period strongly believe either that $s=H$ or that $s=L$. This strong belief results in less overall experimentation in the second period, than under an ASG.

With no dynamics in the last period of the model, the planner uses the same cutoff belief of 0.5 that firms use under an ASG. Under this policy, the planner continues to underexperiment relative to firms in an ASG (see table 2).

Summing over all three periods, the planner induces a higher measure of firms to adopt the stochastic technology. In addition, by having significantly more firms adopt in the first period, the planner increases the odds that the public signal will be more informative in the initial period. When the public signal is uninformative, however, the generation of information under the planner results in firms experimenting less in later periods of the model relative the ASG equilibrium.

The remainder of this paper formally describes the model.

\section{The Model}

In this section, I formally describe the environment of the model and state the firm's technology-adoption problem. I then define an equilibrium.

\subsection{The Environment}

Given the environment in the example, the following generalities apply. The model has $T$ periods, and the probability distribution over $\mu$ is

1. $P(\bar{\mu} \mid s=H)=\psi$,

2. $P(\underline{\mu} \mid s=L)=\psi$, and 
3. $0.5<\psi<1$

where $P(A)$ is the probability of the occurrence of event $A$ occurring. These assumptions imply that when $s=H$, a firm is more likely to produce $\bar{\mu}$ than $\underline{\mu}$. In defining $Y_{s}=$ $\sum_{\mu \in\{\underline{\mu}, \bar{\mu}\}} \mu \cdot P(\mu \mid s)$, I assume values of $\underline{\mu}$ and $\bar{\mu}$ such that

4. $Y_{L}<\bar{Y}<Y_{H}$, and

5. $\left(\bar{Y}-Y_{L}\right)=\left(Y_{H}-\bar{Y}\right)$.

Thus the stochastic technology has an expected output higher than that of the safe technology only if $s=H$. The symmetry simplifies solving the model.

Aggregate uncertainty exists in this environment because the parameter $s$ is unknown. Firms have priors, denoted $\gamma$, over $s \in S$. As $S$ is a two-element set, I let $\gamma=P(s=H)$. The aggregate distribution of firms' beliefs is denoted $\delta$, and the distribution of firms' beliefs conditional on $s$ is $\delta_{s}$.

\subsection{The Firm's Problem}

The firm's problem is to maximize total discounted quantity produced over $T$ periods. In each period, firms make their adoption decisions, produce output, and then receive their private and public signals. In all but the last period, learning has value in as much as it provides information about the stochastic technology that is useful for future adoption decisions. In this section, I first explain how firms learn and then describe the firm's dynamic technology adoption problem.

\subsubsection{Learning}

The central feature of the firm's problem is in updating the firm's beliefs about the parameter $s$. As detailed earlier, firms learn by using the risky technology and by observing a public signal of aggregate quantity.

To model how a firm learns in this environment, I use a variation of the ASG framework. Firms begin the model knowing the initial distribution of aggregate beliefs, $\hat{\delta}$, which I assume is a point mass. Other firms' beliefs and actions are inputs into the firm's 
problem, as they influence the strength of the public signal. Over the course of the model, firms observe a sequence of public signals and receive an individual sequence of private signals. To track the evolution of the aggregate distribution of beliefs, a firm needs to infer which firms are experimenting with the stochastic technology. Let the distributional strategy $\tau_{t}\left(\gamma ; \hat{\delta}, \tau^{t}, \tilde{Q}^{t}\right) \in[0,1]$ provide such a description, where $\tau^{t}=\left\{\tau_{0}, \tau_{1}, \ldots, \tau_{t-1}\right\}$ and $\tilde{Q}^{t}=\left\{\tilde{Q}_{0}, \tilde{Q}_{1}, \ldots, \tilde{Q}_{t-1}\right\}$. Given the initial distribution of beliefs, the distributional strategy details the adoption strategy of a firm with belief $\gamma$ given the history of past adoption strategies and of past public signals. When $\tau_{t}$ is equal to 0 , the firm's strategy is to adopt the stochastic technology with probability 0 . Two sequences, $\left(\tau^{t}, \tilde{Q}^{t}\right)$, characterize the state space at time $t$, given $\hat{\delta}$. These sequences allow each firm to deduce how the conditional aggregate distributions of firms' beliefs evolve, given the initial distribution of firms' beliefs. Hence, an alternative characterization of the state space is the pair of aggregate distributions of firms' beliefs conditional on $s,\left(\delta_{L}, \delta_{H}\right)$. Naturally, as firms differ on the probability that $s=H$, they disagree on the unconditional distribution of beliefs. Notational, it is simpler to use the pair of conditional distributions of beliefs to describe the state space rather than the sequences of $\tau$ and $\tilde{Q}$. Let $\left\{\delta_{s}\right\}=\left(\delta_{L}, \delta_{H}\right)$ and then re-define the distributional strategy as $\tau\left(\gamma ;\left\{\delta_{s}\right\}\right)$, dropping the dependence on $\hat{\delta}$.

Using the distributional strategy, I can define a firm's expectations of aggregate quantity and so specify how firms learn from the public signal. Aggregate output, conditional on $s$, is given by

$$
Q_{s}\left(\tau_{t},\left\{\delta_{s}\right\}\right)=\bar{Y} \int_{0}^{1}\left[1-\tau_{t}\left(x ;\left\{\delta_{s}\right\}\right)\right] \delta_{s}(x) d x+Y_{s} \int_{0}^{1} \tau_{t}\left(x ;\left\{\delta_{s}\right\}\right) \delta_{s}(x) d x .
$$

This function sums up each firm's expected output to arrive at a deterministic aggregate quantity, conditional on $s$ and $\left(\delta_{L}, \delta_{H}\right)$. The first part of $Q_{s}$ aggregates the output of firms using the deterministic technology, and the second part sums up the expected output of those using the stochastic technology. Conditional on $s$, the large number of firms in the economy ensures that the expected output of the stochastic technology is equal to the actual quantity produced.

With $Q_{s}$ defined, I can describe how beliefs are updated through the public signal. 
Recall that the public signal is defined as

$$
Q^{*}=Q+\varepsilon
$$

where $Q$ denotes actual aggregate quantity and $\varepsilon \sim U(-b, b)$ for $b>0$. Exogenous noise is essential to prevent firms from always learning the true value of $s$ with complete certainty. Without $\varepsilon$, firms could simply invert $Q_{s}\left(\tau_{t},\left\{\delta_{s}\right\}\right)$ and determine the true value of $s .{ }^{5}$ Using the uniform distribution with a finite support simplifies the learning process, as it implies that a firm either learns nothing from the public signal or learns the true value of $s$ completely. As shown in figure 3, the support of $Q^{*}$ differs depending on the true value of $s$, when $Q_{L}\left(\tau_{t},\left\{\delta_{s}\right\}\right) \neq Q_{H}\left(\tau_{t},\left\{\delta_{s}\right\}\right)$. ${ }^{6}$ For values of the public signal that fall in the support of $Q^{*}$ for both values of $s$, a firm learns nothing because these noisy signals are equally likely to have been generated in an economy where $s=L$ as in one where $s=H$. For values of $Q^{*}$ that are possible only given one of the values of $s$, a firm immediately learns the true value of $s$.

The probability that firms learn the true value of $s$ from the public signal is simply the odds of drawing an $\varepsilon$ that produces a $Q^{*}$ that is uniquely identified with a particular underlying value of $s$. This probability is equal to

$$
\tilde{p}\left(\tau_{t},\left\{\delta_{s}\right\}\right)=\frac{Q_{H}\left(\tau_{t},\left\{\delta_{s}\right\}\right)-Q_{L}\left(\tau_{t},\left\{\delta_{s}\right\}\right)}{2 b} .
$$

A firm is more likely to learn the true value of $s$ as the difference in the conditional aggregate quantities increases. Also, as the support of the error term $\varepsilon$ increases or as the signal gets noisier, the probability of learning anything from the public signal decreases. As expectations of $Q_{s}$ do not depend on individual beliefs, if firms agree on $\left(\tau_{t},\left\{\delta_{s}\right\}\right)$, they all have the same probability of learning everything from the public signal.

Private signals inform firms through a learning-by-doing mechanism. The private signals that firms observe are their individual outputs, $\{\underline{\mu}, \bar{Y}, \bar{\mu}\}$. Using Bayes rule, a

\footnotetext{
${ }^{5}$ As equation 4 makes clear, $b>\frac{Q_{H}\left(\tau_{t},\left\{\delta_{s}\right\}\right)-Q_{L}\left(\tau_{t},\left\{\delta_{s}\right\}\right)}{2}$ must hold in order for the noisy component of $Q^{*}$ to prevent a firm from completely learning $s$.

${ }^{6} Q_{H}=Q_{L}$ only if the measure of adopting firms equals zero.
} 
Figure 3: Conditional Support of $Q^{*}$

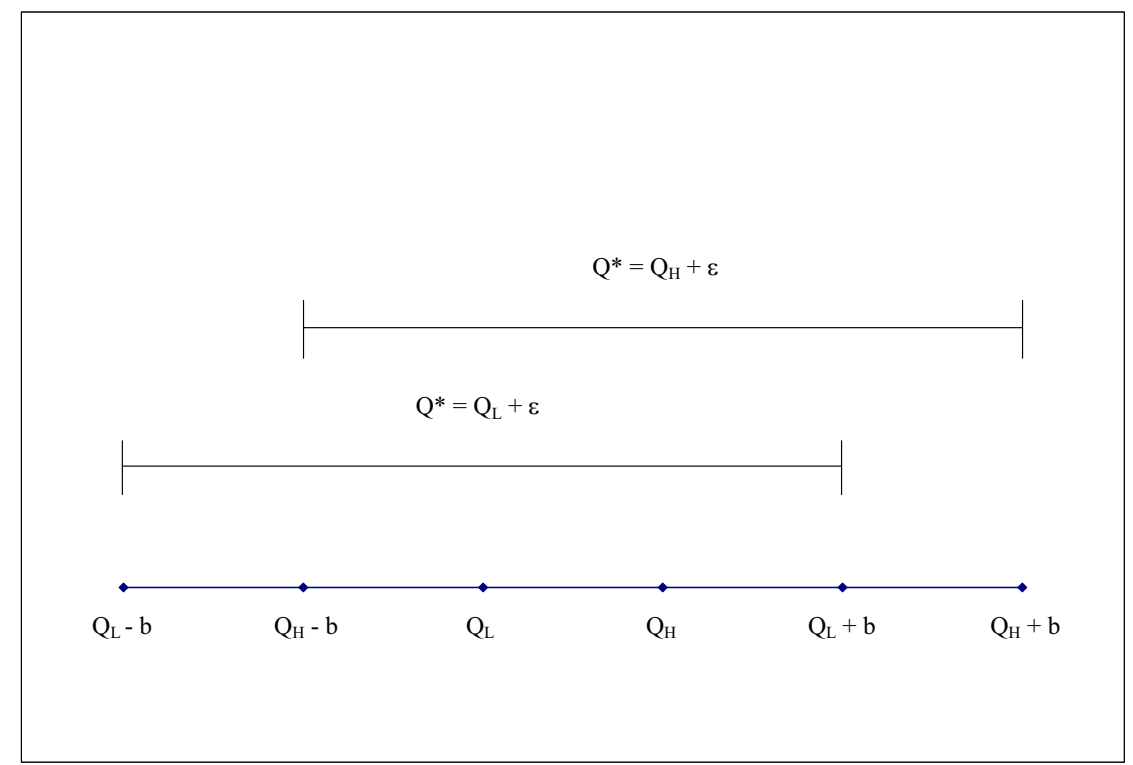

firm's posterior belief, given a prior belief $\gamma$, is

$$
\begin{array}{ll}
\underline{\gamma}(\gamma)=\frac{(1-\psi) \gamma}{(1-\psi) \gamma+\psi(1-\gamma)}, & \text { if observe } \underline{\mu}, \text { or } \\
\bar{\gamma}(\gamma)=\frac{\psi \gamma}{\psi \gamma+(1-\psi)(1-\gamma)}, & \text { if observe } \bar{\mu}, \text { or } \\
\gamma, & \text { if observe } \bar{Y} .
\end{array}
$$

Note that $0 \leq \underline{\gamma} \leq \gamma \leq \bar{\gamma} \leq 1$, where strict inequalities hold for $\gamma \in(0,1)$. Because the safe technology is deterministic, firms that observe $\bar{Y}$ learn nothing about $s$. In cases in which there is no confusion over the prior belief, I suppress the posterior functions' dependence on $\gamma$. 
With the flow of public and private signals of $s$, the conditional distributions of aggregate distributions evolve deterministically. To represent the case in which the public signal is informative, I let $\delta_{0}$ and $\delta_{1}$ denote a point mass of beliefs at $\gamma=0$ and $\gamma=1$ respectively. When the public signal is uninformative, I let $\tilde{\delta}\left(\tau_{t},\left\{\delta_{s}\right\}\right)$ return the pair of posterior conditional distributions of aggregate beliefs, given the distributional strategy and the prior conditional distributions.

\subsubsection{Value Function}

The firm's problem is to pick a strategy over the choice of technologies to use in each period. Its objective is to maximize total discounted output, which involves computing the returns that learning has on future output. The firm's problem is easiest to solve by going backwards. In the last period, $T$, the firm's problem given a belief $\gamma$ is

$$
V_{T}\left(\gamma ; \tau_{T},\{\delta\}\right)=\max _{e \in[0,1]}\left\{(1-e) \bar{Y}+e\left(Y_{H} \gamma+Y_{L}(1-\gamma)\right)\right\} .
$$

If a firm chooses not to use the stochastic technology, that is $e=0$, then it produces $\bar{Y}$ with certainty. If it experiments however, then it expects $Y_{H}$ with probability $\gamma$ and $Y_{L}$ with probability $(1-\gamma)$. As these two state variables influence how a firm learns, the distribution of firms' beliefs and the set of distributional strategies do not matter in the last period of the model. Solving the problem is straightforward and results in firms following a cutoff rule, where only firms who hold a $\gamma>0.5$ adopt the stochastic technology. This cutoff point is a result of the assumptions of symmetry on $\left\{Y_{L}, \bar{Y}, Y_{H}\right\}$. 
For each period except the last, $t=1,2, \ldots, T-1$, the firm's problem is

$$
\begin{aligned}
V_{t}\left(\gamma ; \bar{\tau},\left\{\delta_{s}\right\}\right)= & \max _{e \in[0,1]}\left\{(1-e) \bar{Y}+e\left(Y_{H} \gamma+Y_{L}(1-\gamma)\right)+\right. \\
& \beta\left(\tilde{p}\left(\tau_{t},\left\{\delta_{s}\right\}\right)\left[V_{t+1}\left[1 ; \bar{\tau}, \delta_{1}\right] \gamma+V_{t+1}\left[0 ; \bar{\tau}, \delta_{0}\right](1-\gamma)\right]+\right. \\
& {\left[1-\tilde{p}\left(\tau_{t},\left\{\delta_{s}\right\}\right)\right]\left[(1-e) V_{t+1}\left[\gamma ; \bar{\tau}, \tilde{\delta}\left(\tau_{t},\left\{\delta_{s}\right\}\right)\right]+\right.} \\
& \left.\left.\left.e\left(V_{t+1}\left[\bar{\gamma} ; \bar{\tau}, \tilde{\delta}\left(\tau_{t},\left\{\delta_{s}\right\}\right)\right] \alpha(\gamma)+V_{t+1}\left[\underline{\gamma} ; \bar{\tau}, \tilde{\delta}\left(\tau_{t},\left\{\delta_{s}\right\}\right)\right][1-\alpha(\gamma)]\right)\right]\right)\right\},
\end{aligned}
$$

where $\alpha(\gamma)$ is the probability of observing $\bar{\mu}$ given the firm's belief $\gamma$ and $\bar{\tau}=\left\{\tau_{t}\right\}_{t=1}^{T}$. The first line of equation 7 is the firm's current period output, and the remaining lines are the expected output over the rest of the model horizon. The second line defines the case in which the public signal is informative. This case occurs with probability $\tilde{p}\left(\tau_{t},\left\{\delta_{s}\right\}\right)$, and results in the aggregate distribution of firms' beliefs equaling $\delta_{0}$ (point mass at 0 ) or $\delta_{1}$ (point mass at 1). Using its beliefs, the firm assigns probability $\gamma$ to $s=H$, and $(1-\gamma)$ to $s=L$. The third and fourth lines represent the case in which the public signal is not informative. If the firm chooses not to adopt the stochastic technology, then it learns nothing from its individual output and so will have the same belief in the next period that it had in the current period. In this case, the firm's continuation value is $V_{t+1}\left(\gamma, \bar{\tau}, \tilde{\delta}\left(\tau_{t},\left\{\delta_{s}\right\}\right)\right)$. In contrast, if the firm adopts, then it learns by observing its output and enters the next period either with the posterior belief $\underline{\gamma}$ or with $\bar{\gamma}$. The probability of observing $\bar{\mu}$ is given by $\alpha$ and is a function of $\gamma$.

Because a firm has measure 0, its entry decision has no effect on the evolution of aggregate beliefs. However, the distribution of firms' beliefs and the distributional strategy play an important role in the firm's problem through $\tilde{p}$. When deciding whether to experiment with the stochastic technology, the firm takes into account the probability of learning everything through the public signal. As is clear in equation 7 , a higher $\tilde{p}$ devalues the informational gain from the private signal, making private and public signals substitutable sources of information. 


\subsection{Equilibrium}

The equilibrium notation used in this model is a variation on an anonymous sequential game. ${ }^{7}$ In this variation, for any belief, a firm's optimal effort decision has to match the prediction given by the distributional strategy $\bar{\tau}=\left\{\tau_{t}\right\}_{t=1}^{T}$. To state this equilibrium concept formally, I first define $V_{t}^{e}\left(\gamma ; \bar{\tau},\left\{\delta_{s}\right\}\right)$ as the expected output of a firm in period $t=1,2, \ldots, T$, taking action $e \in[0,1]$, with belief $\gamma$, given the pair $\left\{\delta_{s}\right\}$.

I can then define that a distributional strategy $\bar{\tau}$ is an equilibrium if it satisfies $\forall\left\{\delta_{s}\right\}, \forall e \in[0,1]$, and for $t=1,2, \ldots, T$,

$$
V_{t}^{\tilde{e}_{t}\left(\gamma,\left\{\delta_{s}\right\}\right)}\left(\gamma ; \bar{\tau},\left\{\delta_{s}\right\}\right) \geq V_{t}^{e}\left(\gamma ; \bar{\tau},\left\{\delta_{s}\right\}\right)
$$

where $\tilde{e}\left(\gamma,\left\{\delta_{s}\right\}\right)=\tau_{t}\left(\gamma,\left\{\delta_{s}\right\}\right)$. This condition is an optimality restriction, requiring that $\tau_{t}$ assigns the decision $e$ to a firm that maximizes that firm's objective function, given the state variables.

\section{Analysis of the Firm's Problem}

In this section, I show how the gain from additional information is nonmonotonic in beliefs. I then prove that the optimal policy rule is a cutoff rule.

Intuitively, the firm's problem can be reduced to a comparison between the net present value of the expected output of the firm over the model horizon given that it experiments with the stochastic technology in the current period versus the case where it does not

\footnotetext{
${ }^{7}$ Mitchell (1997) extends the equilibrium concept of Jovanovic and Rosenthal (1988) for economies with aggregate uncertainty, such as the one used in this paper.
} 
experiment and uses the deterministic technology. We know from equation 7 that

$$
\begin{aligned}
V_{t}^{0}\left(\gamma ; \bar{\tau},\left\{\delta_{s}\right\}\right)= & \bar{Y}+\beta \tilde{p}\left(\tau_{t}, \delta\right)\left[V_{t+1}\left(1 ; \bar{\tau}, \delta_{1}\right) \gamma+V_{t+1}\left(0 ; \bar{\tau}, \delta_{0}\right)(1-\gamma)\right]+ \\
& \beta\left[1-\tilde{p}\left(\tau_{t}, \delta\right)\right] V_{n+1}(\gamma ; \bar{\tau}, \tilde{\delta}), \\
V_{t}^{1}\left(\gamma ; \bar{\tau},\left\{\delta_{s}\right\}\right)= & Y_{H} \gamma+Y_{L}(1-\gamma)+\beta \tilde{p}\left(\tau_{t}, \delta\right)\left[V_{t+1}\left(1 ; \bar{\tau}, \delta_{1}\right) \gamma+V_{t+1}\left(0 ; \bar{\tau}, \delta_{0}\right)(1-\gamma)\right]+ \\
& \beta\left[1-\tilde{p}\left(\tau_{t}, \delta\right)\right]\left[V_{t+1}(\bar{\gamma} ; \bar{\tau}, \tilde{\delta}) \alpha(\gamma)+V_{t+1}\left(\underline{\gamma} ; \tau_{t}, \tilde{\delta}\right)[1-\alpha(\gamma)]\right],
\end{aligned}
$$

where $\tilde{\delta}^{\prime}$ 's dependence on $\tau_{t}$ and $\left\{\delta_{s}\right\}$ have been suppressed. Subtracting $V_{t}^{1}-V_{t}^{0}$ results in the indifference condition

$$
\begin{aligned}
& 0=Y_{H} \gamma+Y_{L}(1-\gamma)-\bar{Y}+ \\
& \quad \beta\left[1-\tilde{p}\left(\tau_{t}, \delta\right)\right]\left[V_{t+1}(\bar{\gamma} ; \bar{\tau}, \tilde{\delta}) \alpha(\gamma)+V_{t+1}\left(\underline{\gamma} ; \tau_{t}, \tilde{\delta}\right)[1-\alpha(\gamma)]-V_{t+1}(\gamma ; \bar{\tau}, \tilde{\delta})\right] .
\end{aligned}
$$

The first line of the indifference condition compares the return on current period output from experimentation and the second line compares the future return. By assumption, the term in the first line is negative for $\gamma=0$ and positive for $\gamma=1$, and has a positive, linear slope in $\gamma$. Because of the properties of Bayes rule, the second line is non-monotonic. For $\gamma \in\{0,1\}, \bar{\gamma}=\gamma=\underline{\gamma}$ and so the second line is equal to zero at these two points. For intermediate values of $\gamma$, as $\underline{\gamma}<\gamma<\bar{\gamma}$, the value of the second line depends on the curvature of $V$. The following lemma proves that $V$ is strictly convex, which, by Jensen's inequality, implies that the second line is strictly positive for all $\gamma \in(0,1)$. Thus, private signals are valuable as they increase future expected output, but the informational gain is nonmonotonic in $\gamma$.

Lemma 1. For all $n=1,2, \ldots, N, V_{t}$ is increasing and strictly convex in $\gamma$.

Proof. Through induction, it is easy to show that $V_{t}$ is increasing in $\gamma$. Similarly, I use induction to show that $V_{t}$ is strictly convex in $\gamma$. I prove convexity using the fact that $V_{t}=\max \left\{V_{t}^{0}, V_{t}^{1}\right\}$ and that $V_{T}$ is strictly convex. Because $V_{t-1}^{0}$ is a linear transformation of $V_{t}$, it is convex. In addition, dropping the dependence on $\left(\tau,\left\{\delta_{s}\right\}\right)$, and letting $F_{t}(\gamma)=$ 
$V_{t}(\bar{\gamma}) \alpha(\gamma)+V_{t}(\underline{\gamma})[1-\alpha(\gamma)]$, shows that $V_{t-1}^{1}$ is strictly convex for all $t=1,2, \ldots, T$, as,

$$
\begin{array}{r}
F_{t}(\bar{\gamma}) \alpha(\gamma)+F_{t}(\underline{\gamma})[1-\alpha(\gamma)]> \\
V_{t}(\bar{\gamma}) \alpha(\gamma)+V_{t}(\underline{\gamma})[1-\alpha(\gamma)]= \\
F_{t}(\gamma)
\end{array}
$$

Despite the nonmonotonicity of the return to receiving a private signal, the optimal policy rule for firms remains a cutoff rule. Theorem 1 proves this result.

Theorem 1. Under an anonymous sequential game, the distribution strategy $\bar{\tau}$ is a cutoff rule. Hence for every $\left\{\delta_{s}\right\}$ and $t=1,2, \ldots, T$, there exists a cutoff belief $\hat{\gamma}$ such that

$$
\begin{aligned}
& \tau_{t}\left(\gamma,\left\{\delta_{s}\right\}\right)=0 \text { for all } \gamma<\hat{\gamma}, \text { and } \\
& \tau_{t}\left(\gamma,\left\{\delta_{s}\right\}\right)=1 \text { for all } \gamma>\hat{\gamma}
\end{aligned}
$$

Proof. See appendix A.2 for details of the proof.

This result is in line with the results in classic two-armed bandit problems, in which information is also nonmonotonic in beliefs (see Jensen (1983) and Bolton and Harris (1999), for example).

Now that I have defined the firm's problem and can solve for the optimal policy rule under an ASG, I consider the efficiency of the rate of adoption. Naturally, to evaluate the speed with which firms adopt the stochastic technology, I need to solve for the rate of adoption under a social planner. The next section details the planner's problem.

\section{Social Planner}

In this section, I define the planner's problem, prove that a cutoff rule solves the planner's problem, and compare this solution to the policy rule under an ASG. I show that the standard result holds: Firms underexperiment relative to the planner in the current 
period, ceteris paribus. I then examine the sequential generation of information under a planner.

\subsection{The Social Planner's Problem}

As detailed earlier, the standard social-planner problem of maximizing aggregate quantity is not applicable to this model. Therefore, I construct an uninformed planner's problem in which the planner, who has no information himself, acts as a simple coordinating device and specifies strategies for agents as a function of their own beliefs. The planner's objective is to implement a distributional strategy that results in a Paretooptimal outcome, where firms are not worse off relative to the outcome from an ASG. I let $\bar{\tau}$ be the distributional strategy in an ASG, denote $\nu_{t}$ as the planner's distributional strategy in period $t$, and let $\bar{\nu}=\left\{\nu_{t}\right\}_{t=1}^{T}$. Then the planner's problem is to choose the $\nu_{t}$ that solves, for all $\gamma$,

$$
\begin{aligned}
V_{t}\left(\gamma ; \bar{\nu},\left\{\delta_{s}\right\}\right)= & \max _{\nu_{t}}\left\{\left[1-\nu_{t}(\gamma)\right] \bar{Y}+\nu_{t}(\gamma)\left[Y_{H} \gamma+Y_{L}(1-\gamma)\right]+\right. \\
& \beta \tilde{p}\left(\nu_{t},\left\{\delta_{s}\right\}\right)\left[V_{t+1}\left(1 ; \bar{\nu}, \delta_{1}\right) \gamma+V_{t+1}\left(0 ; \bar{\nu}, \delta_{0}\right)(1-\gamma)\right]+ \\
& \left.\beta\left[1-\tilde{p}\left(\nu_{t},\left\{\delta_{s}\right\}\right)\right] E_{\nu_{t}}\left[V_{t+1}\right]\right\}, \\
\text { s.t. } \quad & V_{t}\left(\gamma ; \bar{\nu},\left\{\delta_{s}\right\}\right) \geq V_{t}\left(\gamma ; \bar{\tau},\left\{\delta_{s}\right\}\right),
\end{aligned}
$$

where

$$
\begin{aligned}
E_{\nu_{t}}\left[V_{t+1}\right]=\left[1-\nu_{t}(\gamma)\right] V_{t+1}[\gamma ; \bar{\nu}, \tilde{\gamma}]+ & \\
& \nu_{t}(\gamma)\left[V_{t+1}(\bar{\gamma} ; \bar{\nu}, \tilde{\delta}) \alpha(\gamma)+V_{t+1}(\underline{\gamma} ; \bar{\nu}, \tilde{\delta})[1-\alpha(\gamma)]\right] .
\end{aligned}
$$

This problem is almost identical to the firm's problem, except that the planner explicitly takes into account how the measure of adopting firms affects the strength of the public signal. As can be seen in the problem above, $\nu_{t}$ appears both in the expected output of the firm and in the argument of $\tilde{p}$. In the firm's problem, the firm took $\tilde{p}$ as given and chose an experimentation strategy. 
By setting $\bar{\nu} \equiv \bar{\tau}$, the planner can mimic the outcome obtained under an ASG. I show, however, that the planner can make all firms better off by inducing more experimentation. Having more firms adopt the stochastic technology benefits all firms through a higher $\tilde{p}$, even those marginal firms that otherwise would not adopt under an ASG.

The following theorem proves that any solution to the social planner's problem is a cutoff rule.

Theorem 2. Any solution to the planner's problem is a cutoff rule $\hat{\nu}$, such that all firms with beliefs greater than $\hat{\nu}$ enter with probability 1 and all firms with beliefs less than $\hat{\nu}$ enter with probability 0. Firms holding the cutoff rule employ a mixed strategy.

Proof. The social planner's problem can be divided into two stages. First, the planner picks the measure of firms that will adopt the risky technology. Conditional on this measure of entry, the planner then chooses the strategy that maximizes firms' expected output, such that each firm is weakly better off relative to the outcome in an ASG. From the proof of theorem 1, we know that cutoff rules maximize firm's expected output. Hence, for any measure of entry that the planner implements, a cutoff rule is the optimal strategy.

Unlike in an ASG, typically there is not a unique solution to the social planner's problem. However, given that cutoff rules solve the planner's problem, I can compare the cutoff rules that solve the planner's problem with the distributional strategies in an ASG. The following proposition shows that the planner, in the current period, always induces a weakly greater measure of firms to experiment relative to the outcome in an ASG, ceteris paribus. The planner experiments strictly more for "interior" solutions. Within this environment, I define an interior solution as one where the cutoff belief is a mass point in the distribution of firms' beliefs and those firms at the cutoff belief employ a mixed strategy.

Proposition 1. Compared with the measure of adopting firms under an ASG, a social planner chooses a weakly higher level of experimentation in the current period, given the same set of state variables. Further, given that $\tau_{t}$ is an interior solution to the firm's problem under an ASG, all cutoff rules that solve the equivalent planner's problem are 
strictly less than $\tau_{t}$. Hence, for interior solutions, the planner induces a strictly higher level of experimentation relative to the measure of adopting firms under an $A S G$.

Proof. Because $\tilde{p}$ is an increasing function of the measure of firms that adopt the stochastic technology, the planner must have at least the same measure of firms experiment as occurs in an ASG. This implies that any cutoff rule used by the planner either has a lower cutoff belief, or employs the same cutoff belief but uses a weakly higher probability of entry compared with the cutoff rule in an ASG.

I now show that, given that $\tau_{t}$ is an interior solution, the planner has a strictly higher measure of firms experiment with the stochastic technology. Because $\tau_{t}$ is an interior solution, I can evaluate the derivative of $V_{t}$ from the planner's problem at the cutoff belief. If $\hat{\gamma}$ denotes the cutoff belief, then the derivative is

$$
\begin{aligned}
\frac{d V_{t}}{d \nu_{t}}= & -\bar{Y}+Y_{H} \gamma+Y_{L}(1-\gamma)+ \\
& \beta \frac{d \tilde{p}}{d \nu_{t}}\left[V_{t+1}\left(1 ; \bar{\nu}, \delta_{1}\right) \gamma+V_{t+1}\left(0 ; \bar{\nu}, \delta_{0}\right)(1-\gamma)-E_{\nu_{t}}\left[V_{t+1}\right]\right]+ \\
& \beta\left[1-\tilde{p}\left(\nu_{t},\left\{\delta_{s}\right\}\right)\right] \frac{d E_{\nu_{t}}\left[V_{t+1}\right]}{d \nu_{t}} .
\end{aligned}
$$

Because of the envelope condition, the first and third lines of this derivative are equal to the firm's indifference condition, shown by equation 10. We know that in an ASG the firm holding the cutoff belief is indifferent between the two technologies. The second line of equation 14, the return from the public signal, is negative, as $\frac{d \tilde{p}}{d \nu_{t}}<0$ and the term in the brackets is positive for $\gamma \in(0,1)$. Thus for the marginal firm under an ASG, $\left.\frac{d V_{t}}{d \tau_{t}}\right|_{\hat{\gamma}}<0$. Hence the optimal cutoff point for the planner is strictly less than $\hat{\gamma}$, the cutoff belief under an ASG.

This result is in line with the literature and not unusual given the externalities associated with the public signal. Its importance lies in showing that all the optimal cutoff rules a planner might implement are weakly less than $\tau_{t}$. This proposition also has significant implications for the generation of information within the economy. In the first period of the model, the state variables, or initial conditions, are the same for the planner's problem and for the firms in an ASG. By proposition 1, we know that, except in the case 
of corner solutions, the planner will induce a higher level of adoption than will firms in a market equilibrium. This difference in strategies implies not only that $\tilde{p}$ is higher under a planner but also that firms receive more private signals. As shown in the example at the beginning of the paper, this faster generation of information is particularly significant in that it lowers the return on information in later periods of the model. In certain cases, this devaluation of information leads to the planner underadopting relative to firms in the corresponding ASG. The following proposition formally states this result:

Proposition 2. Relative to an uninformed social planner, firms do not always underadopt the stochastic technology in all periods. Rather, parameters values exist such that firms overadopt the risky technology in each period of the model except the first.

The three-period model considered earlier provides an example of this underadoption result, which stands in contrast to the literature. In Rob (1991), the planner always weakly experiments more than firms do in a market equilibrium. Further, Bolton and Harris (1999) show that firms working as a team experiment to a larger extent than firms acting individually at any point in time. This proposition builds upon these results by demonstrating that firms underexperiment relative to the planner in the initial period and when summing over all periods of the model. This underadoption result, however, does not necessarily hold in each period of the model.

\section{Conclusion}

In this paper I examine the speed of adoption by firms in an environment with private and public signals. The standard result holds: All else being equal, the planner chooses a higher level of experimentation relative to firms in a market equilibrium. More interesting, I study the generation of information over the model's lifetime. I find that the planner seeks to exploit the gains of experimenting earlier and so generates more information in the initial periods of the model. Generating more private signals in the beginning of the model results in firms decreasing the value of additional information later in the model. Hence firms, under a planner, might underadopt relative to firms in a market equilibrium in the latter periods of the model. 


\section{A Appendix}

\section{A.1 Computational Details}

This section of the appendix details the computational techniques used to solve both the firm's problem and the planner's problem. I then show why the solution to the planner's problem in the last period is unique.

The large dimension of the second period's state space, all possible $\left\{\delta_{s}\right\}$, makes implementing the standard backward induction algorithm difficult. Therefore, I solve for the optimal adoption strategies under an ASG equilibrium and under an uninformed social planner by using a two-step optimization algorithm.

I first explain this algorithm within the context of solving the model as an ASG. In the third period of the model, the static nature of the firm's problem implies that the optimal cutoff rule is one-half. In the second period, a bisection routine can be used to solve for the optimal cutoff rule by using the firm's indifference condition, equation 10 , for a given $\left\{\delta_{s}\right\}$. The two relevant conditional distributions of firms' beliefs in the second period, however, depend upon firms' adoption strategies in the first period. Because the optimal adoption strategy in the first period depends on adoption strategies in the second period, I employ a pair of bisection routines to compute the optimal adoption strategies in the first and second periods. The outer bisection routine solves the firm's indifference condition in the first period. To compute firms' expected utility in the second and third periods, however, this outer routine calls the second bisection routine. This second, inner routine solves for the optimal cutoff belief in the second period, given a guess of the policy rule in the first period. In this manner, I simultaneously numerically solve for the optimal cutoff rules in the first and second periods of the model in an ASG.

I use a similar algorithm to solve the planner's problem. The planner's problem has an additional twist as there are multiple solutions. I choose to find the optimal cutoff belief that is closest to the cutoff rule used in an ASG. To find this particular solution to the planner's problem, I once again use a two-step optimization routine. In place of the bisection routines, however, I employ an iterative search over the set of possible cutoff beliefs and mixed strategies. This iterative search starts with a cutoff belief of one-half 
and a mixed strategy in which no firm holding cutoff belief enters. ${ }^{8}$ Every firm's expected utility is computed under this policy rule. Then, I consider a policy rule that involves slightly more firms experimenting. If a mass of firms exist at the cutoff point, I increase the probability of those firms entering. Otherwise, I lower the cutoff belief to the next lowest mass point of firms' beliefs and consider a low probability of adoption. Firms' expected utilities are then re-evaluated and compared with the results under the previous cutoff rule. If all firms are weakly better off, this new policy rule Pareto dominates the previous one. I then try a policy rule with even more experimentation. At some point, the outcome under the new policy rule will not Pareto dominate the previous outcome. When this occurs, the previous policy rule must generate a Pareto-optimal outcome. By construction, it is not Pareto dominated by the outcome associated with a policy rule with a higher cutoff belief. Further, from the proof of theorem 1, we know that if an outcome associated with a rule is not Pareto dominated by the outcome generated by a cutoff belief slightly below it, then this outcome is not Pareto dominated by any outcome generated by policy rules with lower cutoff beliefs. ${ }^{9}$ This policy rule is also the "highest" cutoff rule that solves the planner's problem, or is the one closest to the outcome under an ASG. To check that I solved for the correct policy rule under the planner, I compare each firm's outcome under each regime and check that the planner's rule induces weakly more adoption and that each firm under the planner has weakly higher expected utility.

In the three-period example, the planner's policy rule is unique. Given the solution algorithm I used, other possible policy rules must use cutoff beliefs below the ones I reported. This is not possible in the first period, where all firms adopt (a corner solution). Further, in the second period, inducing any positive measure of firms with the lower belief to adopt does not satisfy the planner's constraint that the planner's rule Pareto dominate the equivalent ASG outcome. Hence, the reported solutions to the planner's problem in the example are unique.

\footnotetext{
${ }^{8}$ It is easy to show that the cutoff rule in ASG is strictly less than one-half in all periods except the last. So by proposition 1 we know that all solutions to the planner's problem are less than one-half.

${ }^{9}$ This result follows from the fact that as $\gamma$ approaches zero, the expectation of current period output under the risky technology falls. In addition, the return from learning-by-doing falls as more firms enter, decreasing the future output gains from experimenting.
} 


\section{A.2 Proof of Theorem 1}

Proof. This is a proof by induction that closely follows the proof laid out for the first theorem in Jensen (1983). In this proof, I suppress functions' dependence on $\left(\bar{\tau},\left\{\delta_{s}\right\}\right)$. Referring to the notation laid out in equation 9, I know that the assumptions on the production technologies imply that $\forall t=1,2, \ldots T$,

$$
\begin{aligned}
& V_{t}^{0}(0)>V_{t}^{1}(0), \\
& V_{t}^{1}(1)>V_{t}^{0}(1) .
\end{aligned}
$$

Let $D_{t}(\gamma)=V_{t}^{1}(\gamma)-V_{t}^{0}(\gamma)$. To prove the theorem, it suffices to show that $D_{t}$ is strictly increasing in $\gamma$ for all $t$. I know that $D_{T}(\gamma)=Y_{H} \gamma+Y_{L}(1-\gamma)-\bar{Y}$, which is strictly increasing in $\gamma$. By induction, I assume that $D_{t+1}$ is strictly increasing in $\gamma$. Further, for $t=1,2, \ldots T-1$, define $V_{t}^{\{1,0\}}$ as the expected value of experimenting in the current period, not experimenting in the next period, and then continuing optimally thereafter. Let $V_{t}^{\{0,1\}}$ be similarly defined. Finally, let $D_{t}^{1}=V_{t}^{\{1,0\}}-V_{t}^{1}$ and $D_{t}^{0}=V_{t}^{\{0,1\}}-V_{t}^{0}$. It is straightforward to show that $V_{t}^{\{1,0\}}(\gamma)-V_{t}^{\{0,1\}}(\gamma)=(1-\beta) D_{T}(\gamma)$ using the properties of Bayes rule. Then I can show that

$$
D_{t}(\gamma)=D_{t}^{0}(\gamma)-D_{t}^{1}(\gamma)+(1-\beta) D_{T}(\gamma)
$$

where I know that $D_{T}$ is strictly increasing in $\gamma$. Turning to the first two terms, I know that

$$
\begin{aligned}
D_{t}^{1}(\gamma) & =V_{t}^{\{1,0\}}(\gamma)-V_{t}^{1}, \\
& =\beta\left(V_{t+1}^{0}-V_{t+1}\right)
\end{aligned}
$$

as the current-period output in both terms is the same. Depending on the private signal received, the right-hand-side equals either 0 or $\beta\left(V_{t+1}^{0}-V_{t+1}^{1}\right)$. By induction, $V_{t+1}^{0}(\gamma)-V_{t+1}^{1}(\gamma)$ is decreasing in $\gamma$. Similarly, it is easy to show that $D_{t}^{0}(\gamma)$ is increasing in $\gamma$. Hence, $D_{t}$ is strictly increasing in $\gamma$. 


\section{References}

BanerJee, A. (1992): "A Simple Model of Herd Behaviour," Quarterly Journal of Economics, 107, 797-817.

Berry, D., And B. Fristedt (1985): Bandit Problems. Chapman and Hall, New York.

Bikhchandani, S., D. Hirshleifer, and I. Welch (1992): "A Theory of Fads, Fashion, Custom and Cultural Change as Informational Cascades," Journal of Political Economy, 100, 992-1026.

Bolton, P., And C. Harris (1999): "Strategic Experimentation," Econometrica, 67, 349-374.

DasGupta, A. (2002): "Coordination, Learning, and Delay," FMG Discussion Paper No. 494.

Jensen, R. (1983): "Innovation Adoption and Diffusion When There are Competing Innovations," Journal of Economic Theory, 29, 161-171.

Jovanovic, B., and R. Rosenthal (1988): "Anonymous Sequential Games," Journal of Mathematical Economics, 17, 77-87.

Mitchell, M. (1997): "Bayesian Learning from Others in Competitive Equilibrium," University of Minnesota mimeo.

RoB, R. (1991): "Learning and Capacity Expansion under Demand Uncertainty," Review of Economic Studies, 58, 655-675.

Rothschild, M. (1974): "A Two-Armed Bandit Theory of Market Pricing," Journal of Economic Theory, 9, 185-202. 